\title{
SPEAKER TRACKING IN REVERBERANT ENVIRONMENTS USING MULTIPLE DIRECTIONS OF ARRIVAL
}

\author{
Christine Evers ${ }^{\circ}$ Boaz Rafaely*, and Patrick A. Naylor ${ }^{\circ}$ \\ - Imperial College London, Dept. Electrical \& Electronic Engineering, SW7 2AZ, London, UK \\ * Ben-Gurion University of the Negev, Dept. Electrical \& Computer Engineering, Beer-Sheva 84105, Israel
}

\begin{abstract}
Accurate estimation of the Direction of Arrival (DOA) of a sound source is an important prerequisite for a wide range of acoustic signal processing applications. However, in enclosed environments, early reflections and late reverberation often lead to localization errors. Recent work demonstrated that improved robustness against reverberation can be achieved by clustering only the DOAs from direct-path bins in the short-term Fourier transform of a speech signal of several seconds duration from a static talker. Nevertheless, for moving talkers, short blocks of at most several hundred milliseconds are required to capture the spatio-temporal variation of the source direction. Processing of short blocks of data in reverberant environment can lead to clusters whose centroids correspond to spurious DOAs away from the source direction. We therefore propose in this paper a novel multi-detection source tracking approach that estimates the smoothed trajectory of the source DOAs. Results for realistic room simulations validate the proposed approach and demonstrate significant improvements in estimation accuracy compared to single-detection tracking.
\end{abstract}

Index Terms - Bayes methods; Direction-of-arrival estimation; Motion estimation; Speech processing.

\section{INTRODUCTION}

Localization of sound sources, such as human talkers, is a crucial prerequisite for equipping machines with spatial awareness of the acoustic environment. Localization of source Directions-ofArrival (DOAs) therefore benefits a wide selection of applications, such as Acoustic Simultaneous Localization and Mapping (aSLAM) [1], speaker diarization [2], and spatial filtering [3].

However, in realistic, enclosed environments, reverberation causes localization errors. Dominant early reflections can lead to false detections of sound sources, whilst late reverberation causes degraded DOA accuracy in classical approaches, such as Estimation of Signal Parameters via Rotational Invariance Techniques (ESPRIT) or Multiple Signal Classification (MUSIC). For increased robustness against late reverberation, [4] proposes to perform MUSIC in the Spherical Harmonic Domain (SHD) only on Time-Frequency (TF)-bins containing the contribution of a single static source, identified using the Direct-Path Dominance (DPD)test. A single DOA estimate is obtained by firstly extracting the maximum peak in each MUSIC pseudo-spectrum, and secondly averaging the results across time and frequency. However, spurious peaks due to dominant early reflections cause errors in the averaging process. For improved robustness against early reflections,

The research leading to these results has received funding from the European Unions Seventh Framework Programme (FP7/2007-2013) under grant agreement no. 609465 .
[5] therefore proposes to fit a Gaussian Mixture Model (GMM) to all pseudospectra that passed the DPD-test in order to cluster, and hence separate, the DOAs due to the direct path and other DOAs. Due to speech inactivity and early reflections, some or all Gaussian Mixture (GM) components may correspond to directions away from the direct path. As the source may be detected by several GM components, a single DOA estimate of the source is obtained as the mean of the Gaussian with maximum Component Proportion (CP).

Whilst $[4,5]$ focus on static sources, human talkers are naturally highly dynamic. Therefore, DOAs need to be estimated over short time frames to capture the time-variation in the spatially diverse source directions. However, due to sparsity of speech in time and frequency, early reflections and reverberation can dominate the short-term speech spectrum, and therefore lead to limited statistics and failure of the GMM clustering. To overcome resulting outliers, target tracking $[6,7]$ can be used to estimate a smoothed trajectory of the source direction. However, by relying only on the Gaussian with maximum $\mathrm{CP}$, valuable information about the source direction is disregarded that may be contained in remaining Gaussians.

The hypothesis of this paper is that spurious DOA estimates due to dominant reflections can be overcome by exploiting constructively all clustered Gaussians. Rather than extracting the single mean of the maximum GM component as the estimated DOA, all available GM components are used to propagate the source trajectory. The proposed tracking approach explicitly models DOAs away from the direct path as spurious, uniformly distributed false detections, whereas temporal models are exploited to capture the source dynamics. Within a Bayesian framework, the resulting posterior Probability Density Function (pdf) of the source DOA is estimated sequentially using a particle filter. The source DOA is therefore predicted in time by drawing random variates from the dynamical model. The stochastic weight of each sample is obtained by evaluating all combinations of hypotheses that each DOA may be either due to a direct-path or a false detection. Results using realistic room simulations demonstrate improvements in estimation accuracy of $16.55^{\circ}$ and $7.37^{\circ}$ in azimuth and inclination respectively compared to the mean of the maximum GM component.

This paper is structured as follows: Section 2 provides the problem formulation and system model. The proposed tracking approach is detailed in Section 3. Results are presented in Section 4 and conclusions drawn in Section 5.

\section{PROBLEM FORMULATION}

\subsection{Source Dynamics}

The state of the source direction, $\mathbf{s}(t) \triangleq\left[\phi_{t}, \theta_{t}, \dot{\phi}_{t}, \dot{\theta}_{t}\right]^{T}$ is defined by the source azimuth, $\phi_{t}$, elevation, $\theta_{t}$, and corresponding veloci- 
ties, $\dot{\phi}_{t}$ and $\dot{\theta}_{t}$. The source state is modelled as

$$
\mathbf{s}(t)=\vartheta(\boldsymbol{F}(t) \mathbf{s}(t)+\mathbf{r}(t)),
$$

where $\boldsymbol{F}(t)$ models the source dynamics and $\mathbf{r}(t)$ is the zero-mean process noise with covariance, $\boldsymbol{Q}(t)$, and $\vartheta(\alpha)=\bmod (\alpha, 2 \pi)$ captures the angular wrapping around $2 \pi$. In this paper the source dynamics are modelled a constant-velocity model:

$$
\boldsymbol{F}(t) \triangleq\left[\begin{array}{cc}
\boldsymbol{I}_{2} & \Delta_{t} \boldsymbol{I}_{2} \\
\mathbf{0}_{2 \times 2} & \boldsymbol{I}_{2}
\end{array}\right]
$$

where $\Delta_{t}$ is the time delay between time step $t-1$ and $t$, and $\boldsymbol{I}_{2}$ and $\mathbf{0}_{2 \times 2}$ denote the $2 \times 2$ identity and zero-matrix respectively.

\subsection{Signal Model}

The speech signal emitted by the source and impinging on the microphone array can be divided into a sliding window of time frames, such that signals are processed in the Short Time Fourier Transform (STFT) domain. In each time frame, the received signal corresponds to a mixture of noise with the convolution of the source signal and Acoustic Impulse Response (AIR) of the reverberant room. The pressure of the source, $\mathbf{z}(t, k)=\left[z_{1}(t, k), \ldots, z_{Q}(t, k)\right]^{T}$, impinging on the $Q$ microphones at spherical coordinates $\boldsymbol{\Omega}_{q} \triangleq\left[\phi_{q}, \theta_{q}\right]^{T}$ for $q=1, \ldots, Q$ is therefore given in frequency bin, $k \in \mathcal{K}$, and time frame, $t \in \mathcal{T}$, as

$$
\mathbf{z}(t, k)=\mathbf{h}(t, k) s(t, k)+\mathbf{n}(t, k),
$$

where $\mathbf{h}(t, k) \triangleq\left[h_{1}(t, k), \ldots, h_{Q}(t, k)\right]^{T}$ and $h_{q}(t, k)$ is the Acoustic Transfer Function (ATF) between the source and microphone $q \in \mathcal{Q}$, the source signal is given by $s(t, k)$, and $\mathbf{n}(t, k)$ is the $Q \times 1$ sensor noise vector.

By applying the STFT and performing the planewave decomposition [8], the signal can also be represented as the $(N+1)^{2} \times 1$ vector, $\mathbf{a}(t, k) \triangleq\left[a_{00}(t, k), \ldots, a_{N N}(t, k)\right]^{T}$, representing the plane wave density function in the spherical harmonics domain, given by

$$
\mathbf{a}(t, k)=\boldsymbol{Y}^{H}(\boldsymbol{\omega}(t)) \mathbf{s}(t, k)+\tilde{\mathbf{n}}(t, k),
$$

where $\mathbf{s}(t, k)$ is the vector of complex amplitudes of $L$ plane waves at the origin representing the direct sound and room reflections, and $\tilde{\mathbf{n}}(t, k)=\boldsymbol{B}^{-1}(k r)[\boldsymbol{Y}(\boldsymbol{\Omega})]^{-1} \mathbf{n}(t, k)$ corresponds to the transformed noise vector of length $(N+1)^{2}$. Furthermore, $\boldsymbol{Y}(\boldsymbol{\Omega})$ is the $Q \times(N+1)^{2}$ spherical harmonics matrix with the spherical harmonics functions $Y_{n}^{m}\left(\boldsymbol{\Omega}_{q}\right)$ of order $n$ and degree $m$ as its elements at row number $q$ and column number $n^{2}+n+m+1$, and $\boldsymbol{B}(k r)$ is the $(N+1)^{2} \times(N+1)^{2}$ diagonal matrix holding the radial functions that represent the scattering of a plane wave from a rigid sphere. $\boldsymbol{Y}(\boldsymbol{\omega}(t))$ is structured similarly to $\boldsymbol{Y}(\boldsymbol{\Omega})$ for the source arrival directions, $\boldsymbol{\omega}(t)$.

\subsection{DOA estimation using DPD-MUSIC}

A spatial spectrum matrix is composed of vectors a of length $(N+$ $1)^{2}$, defined in (4) by averaging over time and frequency,

$$
\mathbf{R}(t, k)=\overline{\left[\mathbf{a}(t, k) \mathbf{a}^{H}(t, k)\right]},
$$

For each time-frequency bin, a DPD test is applied [4] by computing the ratio between the two largest eigenvalues of matrix $\mathbf{R}$. If this ratio is larger than a predefined threshold, this bin is considered to pass the DPD test. Then, for each bin that passed the DPD test, the number of eigenvalues in the noise subspace are given by the number, $K$, of repeated eigenvalues. The number of sources is therefore given by $N_{t}=Q-K$. The noise subspace is obtained by eigenvectors corresponding to the $K$ repeated eigenvalues.

For each TF bin that passes the DPD test, an estimate, $\boldsymbol{\omega}(t, k)$, of the source Direction-of-Arrival (DoA) can be obtained as the maximum of the MUSIC pseudospectrum, $\boldsymbol{P}(t, k, \boldsymbol{\Theta})$, given by

$$
\boldsymbol{P}(t, k, \boldsymbol{\Theta})=\frac{1}{\left\|\boldsymbol{U}_{n}^{H} \mathbf{y}^{*}(\boldsymbol{\Theta})\right\|^{2}}
$$

where $\mathbf{y}(\Theta)$ is a $(N+1) 2 \times 1$ vector with elements of the spherical harmonics, $Y_{n}^{m}(\boldsymbol{\Theta})$, at column number $n^{2}+n+m+1$ for the search grid of source directions, $\Theta$, and $\boldsymbol{U}_{n}$ is the $(N+1)^{2} \times\left[(N+1)^{2}-1\right]$ noise subspace matrix assuming a single source.

\subsection{GMM fitting of DOAs}

To reduce the effect of dominant reflections, the set of all DOAs that passed the DPD test is clustered into groups using Gaussian mixture modeling, jointly over $\theta$ and $\phi$ [5]. Typically, the dominant Gaussian, i.e. the one with the most significant weight in the mixture, represents that direct sound, while the other Gaussians represent spurious directions. Therefore, the mean of the dominant Gaussian is selected as the source DOA.

However, for short time frames, the DOA corresponding to the dominant GM components may correspond to a direction away from the source due to the effects of reverberation and sparsity of speech. Nevertheless, in time frames where the dominant DOA corresponds to an outlier, the source DOA may be captured by some of the GM components of lower CP.

\section{PROPOSED MULTI-DETECTION TRACKER}

This section proposes to exploit constructively the information of multiple GM components in order to track the source DOA in time.

Using Bayes's theorem, the posterior pdf of the source state, $p\left(\mathbf{s}(t) \mid \boldsymbol{S}^{\prime}, \boldsymbol{\Omega}\right)$, at time $t$ and conditional on the trajectory of DOAs, $\boldsymbol{\Omega} \triangleq\{\boldsymbol{\Omega}(1), \ldots, \boldsymbol{\Omega}(t)\}$ with $\boldsymbol{\Omega}(t) \triangleq\left\{\boldsymbol{\omega}(t, 1), \ldots, \boldsymbol{\omega}\left(t, M_{t}\right)\right\}$, and the history of the source trajectory, $\boldsymbol{S}^{\prime} \triangleq\{\mathbf{s}(0), \ldots, \mathbf{s}(t-1)\}$, is

$$
p\left(\mathbf{s}(t) \mid \boldsymbol{S}^{\prime}, \boldsymbol{\Omega}\right)=\frac{p(\boldsymbol{\Omega}(t) \mid \mathbf{s}(t)) p\left(\mathbf{s}(t) \mid \boldsymbol{S}^{\prime}\right)}{\int p(\boldsymbol{\Omega}(t) \mid \mathbf{s}(t)) p\left(\mathbf{s}(t) \mid \boldsymbol{S}^{\prime}\right) d \mathbf{s}(t)},
$$

where $p(\boldsymbol{\Omega}(t) \mid \mathbf{s}(t))$ is the joint density of detected DOAs and the number of detected reflections, and $p\left(\mathbf{s}(t) \mid \boldsymbol{S}^{\prime}\right)$ the prior pdf, given by probability transformation of (1) by a wrapped Gaussian [9, Chapter 3] distribution, i.e.,

$$
\begin{aligned}
p\left(\mathbf{s}(t) \mid \boldsymbol{S}^{\prime}\right) & =\mathcal{N}^{w}(\mathbf{s}(t) \mid \boldsymbol{F}(t) \mathbf{s}(t-1), \boldsymbol{Q}(t)) \\
& =\sum_{j=-\infty}^{\infty} \mathcal{N}\left(\mathbf{s}(t) \mid g_{j}(\boldsymbol{F}(t) \mathbf{s}(t-1)), \boldsymbol{Q}(t)\right),
\end{aligned}
$$

where $\mathcal{N}^{w}(\cdot)$ denotes the wrapped Gaussian density, and $g_{j}(\mathbf{s}) \triangleq$ $\mathbf{s}-\mathbf{d} j$ and $\mathbf{d} \triangleq[2 \pi, \pi]^{T}$ ensures that the azimuth and inclination component are wrapped around $2 \pi$ and $\pi$ respectively.

\subsection{Likelihood}

As overmodelling can cause GM clusters to split, the source can be detected by none, one, or several GM components. Therefore, any partition of the set of GM components can correspond to the source 

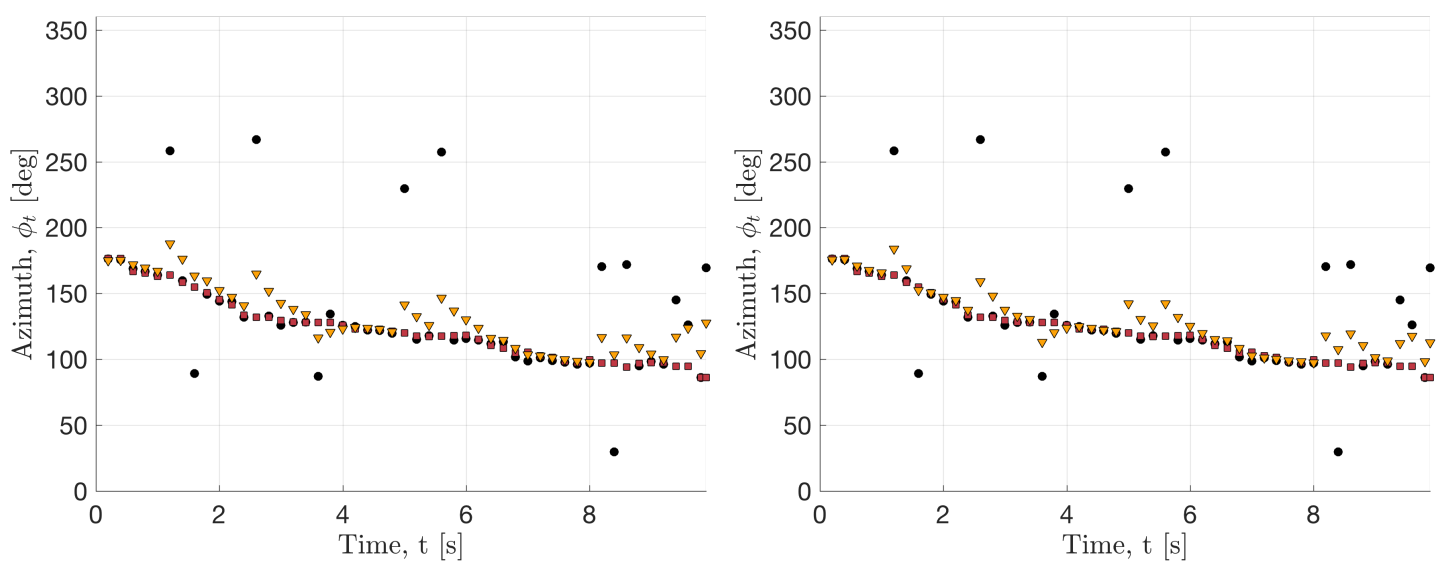

(a) SDPF-noCP

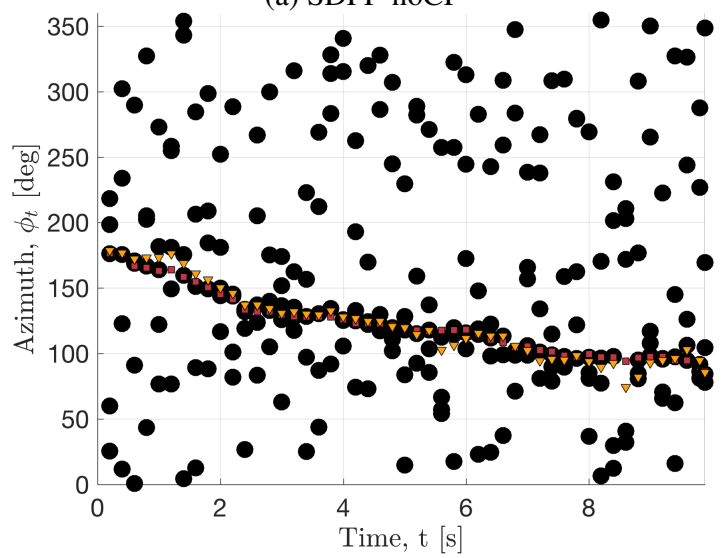

(c) MDPF-noCP

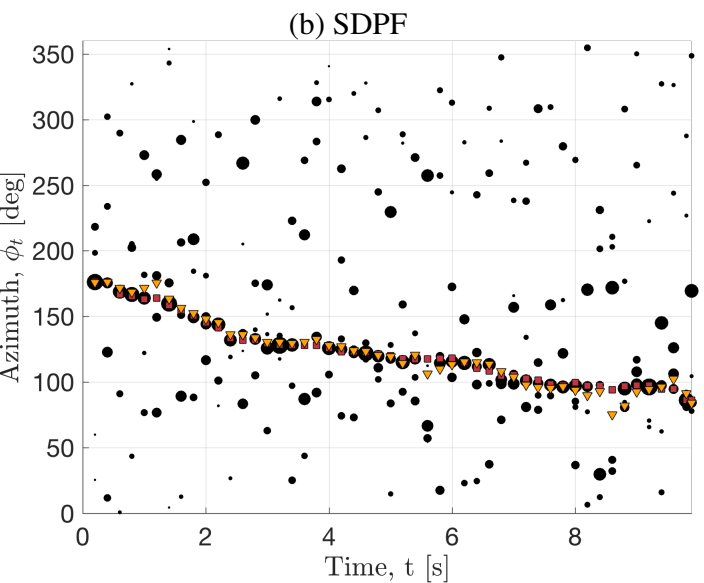

(d) MDPF

Fig. 1: Comparison of true source azimuth (red square) against GM means (black circle) and MDPF estimates (amber triangle).

direction. To account for all possible hypotheses of set partitions, the likelihood of the clustered DOAs is hence described as:

$$
\begin{aligned}
p(\boldsymbol{\Omega}(t) \mid \mathbf{s}(t)) & =\sum_{\boldsymbol{P} \boxminus \boldsymbol{\Omega}(t)} \prod_{\ell \in(\boldsymbol{\Omega}(t)-\boldsymbol{P})} \kappa(\boldsymbol{\omega}(t, \ell)) \prod_{p \in \boldsymbol{P}} p(\boldsymbol{\omega}(t, p) \mid \mathbf{s}(t)),
\end{aligned}
$$

where $p(\boldsymbol{\omega}(t, \ell) \mid \mathbf{s}(t))$ is the single-detection likelihood, $\kappa(\boldsymbol{\omega}(t, \ell)) \triangleq$ $p(\boldsymbol{\omega}(t, \ell) \mid \mathbf{s}(t)=\emptyset)$ is the likelihood of $\boldsymbol{\omega}(t, \ell)$ corresponding to the direction away from the source, and $\boldsymbol{P} \boxminus \boldsymbol{\Omega}(t)$ denotes the nonempty partitions of the set of GM means. E.g., for $M_{t}=3$ with GM means, $\{\boldsymbol{\omega}(t, 1), \boldsymbol{\omega}(t, 2), \boldsymbol{\omega}(t, 3)\}$, the partitions $\boldsymbol{P} \boxminus \boldsymbol{\Omega}(t)$ are given by:

$$
\begin{aligned}
& \{\boldsymbol{\omega}(t, 1), \boldsymbol{\omega}(t, 2), \boldsymbol{\omega}(t, 3)\},\{\boldsymbol{\omega}(t, 1)\},\{\boldsymbol{\omega}(t, 2)\},\{\boldsymbol{\omega}(t, 3)\} \\
& \{\boldsymbol{\omega}(t, 1), \boldsymbol{\omega}(t, 2)\},\{\boldsymbol{\omega}(t, 1), \boldsymbol{\omega}(t, 3)\},\{\boldsymbol{\omega}(t, 2), \boldsymbol{\omega}(t, 3)\}
\end{aligned}
$$

In order to obtain an expression for the measurement likelihood in (9), the GM mean, $\boldsymbol{\omega}(t, \ell)$, in the direction of the source is modelled by the following Markov process:

$$
\boldsymbol{\omega}(t, \ell)=\vartheta(\mathbf{h s}(t)+\mathbf{w}(t, \ell)),
$$

where $\mathbf{h} \triangleq\left[\begin{array}{ll}\boldsymbol{I}_{2} & \mathbf{0}_{2 \times 2}\end{array}\right]$, and $\mathbf{w}(t, \ell) \sim \mathcal{N}\left(\mathbf{0}_{2 \times 1}, \boldsymbol{\Sigma}(t, \ell)\right)$ is the measurement noise with covariance $\boldsymbol{\Sigma}(t, \ell)$. Therefore, the singledetection likelihood, $p(\boldsymbol{\omega}(t, \ell) \mid \mathbf{s}(t))$, in (9) is given by probability transformation of (10) by the wrapped Gaussian density:

$$
p(\boldsymbol{\omega}(t, \ell) \mid \mathbf{s}(t))=\mathcal{N}^{w}(\boldsymbol{\omega}(t, \ell) \mid \mathbf{h} \mathbf{s}(t), \boldsymbol{\Sigma}(t, \ell)) .
$$

The measurement covariance, $\boldsymbol{\Sigma}(t, \ell)$, is unknown in practice. Nevertheless, the GMM provides the weight - or component proportion ,$- \alpha(t, \ell)$, of each Gaussian. We therefore propose utilize the GM as a measure of reliability, such that

$$
\boldsymbol{\Sigma}(t, \ell)=(1-\alpha(t, \ell)) \mathbf{\Psi}
$$

where $\Psi$ is a prior and constant, positive symmetric scale matrix. Furthermore, $\kappa(\boldsymbol{\omega}(t, \ell))$ in (9) is assumed to be uniformly distributed within the room boundaries.

\subsection{Posterior pdf}

As the likelihood in (9) is highly non-Gaussian, the posterior pdf in (7) is analytically intractable. In this paper we thus propose to use a particle filter to approximate the pdf by means of importance sampling [10]. Instead of analytically evaluating (7), the posterior pdf is approximated by a point-mass function of random samples:

$$
p(\mathbf{s}(t) \mid \boldsymbol{\Omega}) \approx \sum_{i=1}^{N} w^{(i)}(t) \delta_{\hat{\mathbf{s}}^{(i)}(t)}(\mathbf{s}(t)),
$$

where $\hat{\mathbf{s}}^{(i)}(t)$ and $w(t)^{(i)}=\frac{\tilde{w}^{(i)}(t)}{\sum_{i=1}^{N} w\left({ }^{(i)} t\right)}$ are the state and normalized weight respectively of particle $i \in \mathcal{N}$ and $\delta_{\hat{\mathbf{s}}^{(i)}(t)}(\mathbf{s}(t))$ is the 


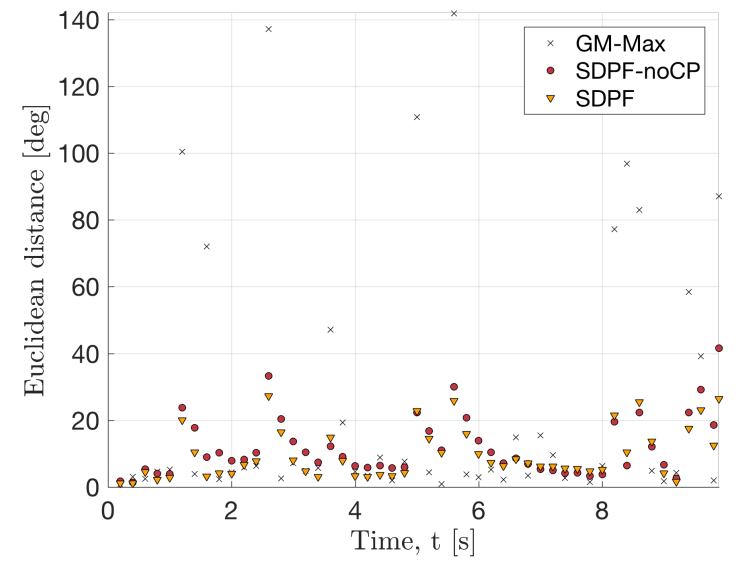

(a) SDPF

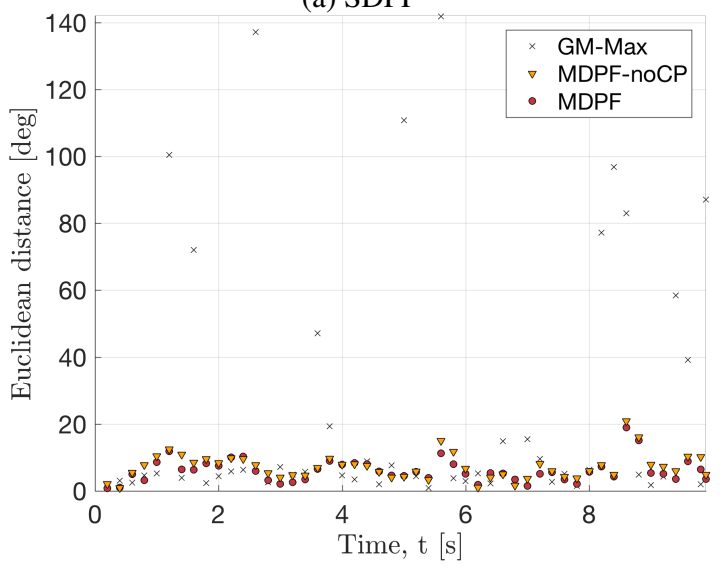

(b) MDPF

Fig. 2: DOA estimation error for the proposed MDPF and the SDPF.

Dirac-delta function of the true source state, evaluated at the random variate, $\hat{\mathbf{s}}^{(i)}(t)$. The particles, $\hat{\mathbf{s}}^{(i)}(t)$, are obtained by sampling from a proposal function - in this paper the prior in (8) - that contains the support of the posterior pdf, $p(\mathbf{s}(t) \mid \boldsymbol{\Omega})$, i.e.,

$$
\hat{\mathbf{s}}^{(i)}(t) \sim p(\mathbf{s}(t) \mid \mathbf{s}(t-1)) .
$$

The unnormalized weights, $\tilde{w}^{(i)}(t)$ are hence given by [10]:

$$
w^{(i)}(t)=w^{(i)}(t-1) p\left(\boldsymbol{\Omega}(t) \mid \hat{\mathbf{s}}^{(i)}(t)\right) .
$$

\section{RESULTS}

In this section, the performance of the proposed algorithm is evaluated for the scenario described in Section 2.3. The impulse response

Table 1: Mean DOA error [deg]

\begin{tabular}{|l||c|c|}
\hline & Azimuth $\phi$ & Inclination $\theta$ \\
\hline \hline GM-Max & $20.72^{\circ}$ & $10.62^{\circ}$ \\
\hline SDPF-noCP & $10.31^{\circ}$ & $4.01^{\circ}$ \\
\hline SDPF & $8.20^{\circ}$ & $3.38^{\circ}$ \\
\hline MDPF-noCP & $4.98^{\circ}$ & $3.93^{\circ}$ \\
\hline MDPF & $4.17^{\circ}$ & $3.25^{\circ}$ \\
\hline
\end{tabular}

of a $6 \times 6 \times 2.5 \mathrm{~m}$ room with $T 60=0.5 \mathrm{~s}$ and sampling frequency of $8 \mathrm{kHz}$ is simulated using the Spherical Microphone array Impulse Response (SMIR) generator [11] for a static 32-channel mh-acoustic eigenmike located at position $(4,2.5,0.54) \mathrm{m}$. The trajectory of a dynamic source is simulated from a Langevin model [12] with an initial position of $(2,2.5,1.8 \mathrm{~m}$ with Root Mean Square (RMS) speed of $0.6 \mathrm{~m} / \mathrm{s}$ and $\beta=2$ with $\Delta_{t}=0.125 \mathrm{~s}$. For each waypoint along the source trajectory, the Room Impulse Response (RIR) between the source and sensor is simulated for a spherical harmonic order of $N=5$ and speed of sound of $343 \mathrm{~m} / \mathrm{s}$. Using the overlap-add approach [13], the RIRs are convolved with a $10 \mathrm{~s}$ anechoic speech signal, created from appended sentences uttered by a randomly selected speaker in the TIMIT database [14]. The reverberant data is divided into blocks of $0.125 \mathrm{~s}$. For each block, the DPD-test is evaluated by applying a STFT with $16 \mathrm{~ms}$ windows. A GMM of 5 components is fit to the set of DOAs that pass the DPD-test.

The proposed MDPF is evaluated using the 5 means of all GM components. The results are compared against using the single mean of the GM component with maximum weight, denoted in the following as the Single-Detection Particle Filter (SDPF). ${ }^{1}$ To evaluate the benefits of constructive exploitation of the GM weights, the MDPF and SDPF are both evaluated using a constant measurement covariance, where $\boldsymbol{\Sigma}(t, \ell)=\boldsymbol{\Psi}$, denoted as MDPF-noCP and SDPFnoCP respectively. For all four cases, the particle filter is evaluated using 200 particles over 10 Monte Carlo runs with $\boldsymbol{Q}(t)=$ $\operatorname{diag}[5 \mathrm{deg}, 1 \mathrm{deg}, 0.1 \mathrm{deg} / \mathrm{s}, 0.1 \mathrm{deg} / \mathrm{s}]^{2}$ and $\boldsymbol{R}(t)=100 \boldsymbol{I}_{2} \mathrm{deg}^{2}$.

The estimated azimuth trajectory is compared against the ground truth and means of the GM components in Fig. 1 for the proposed MDPF against the MDPF-noCP, SDPF, and SDPF-noCP. The SDPF results in Fig. 1a and Fig. 1b indicate that 11 of the GM means correspond to significant outliers, resulting in an average DOA error of $20.72^{\circ}$ in azimuth and $10.62^{\circ}$ in inclination as summarized in Table 1 and Fig. 2 . The outliers cause the tracks to diverge from the ground truth and reconverge after time, resembling a sawtooth behaviour. The SDPF-noCP results in an average DOA error of $10.31^{\circ}$ in azimuth and $4.01^{\circ}$ in inclination. By utilizing the $\mathrm{CP}$ as proposed in (12), the SDPF achieves an improvement of $2.11^{\circ}$ and $0.63^{\circ}$ in azimuth and inclination compared to SDPF-noCP. Furthermore, by exploiting all $5 \mathrm{GM}$ means, the MDPF achieves $4.17^{\circ}$ accuracy in azimuth and $3.25^{\circ}$ in inclination, an improvement of $16.55^{\circ}$ and $7.37^{\circ}$ respectively. The benefit of using the $\mathrm{CP}$ is highlighted by the improvement of the MDPF of $0.81^{\circ}$ in azimuth and $0.68^{\circ}$ in azimuth and inclination compared to the MDPF-noCP.

\section{CONCLUSIONS}

This paper proposed a novel approach to acoustic source tracking using multiple, instantaneous DOAs. A DPD-test is used to identify time-frequency bins that correspond to the contribution of a single source. For each bin that passes the test, MUSIC is used to estimate the corresponding source DOA. The DOAs are clustered in frequency over short blocks of time frames using a GMM. For each block, the clustered DOA, corresponding to the mean of each GM component, are exploited constructively in combination with temporal model of the source dynamics in order to distinguish between spurious and coherent DOAs in order to track the source direction. Results using room simulations for a reverberation time of $500 \mathrm{~ms}$ demonstrate an improvement in estimation accuracy of $16.55^{\circ}$ and $7.37^{\circ}$ in azimuth and inclination respectively.

\footnotetext{
${ }^{1}$ We note that for a single GM mean (9) reduces to a single-detection particle filter [10] with wrapped Gaussian prior and likelihood.
} 


\section{REFERENCES}

[1] C. Evers, A. H. Moore, and P. A. Naylor, "Acoustic simultaneous localization and mapping (a-SLAM) of a moving microphone array and its surrounding speakers," in Proc. IEEE Intl. Conf. on Acoustics, Speech and Signal Processing (ICASSP), Shanghai, China, Mar. 2016.

[2] S. E. Tranter and D. A. Reynolds, "An overview of automatic speaker diarization systems," IEEE Transactions on Audio, Speech, and Language Processing, vol. 14, no. 5, pp. 15571565, Sep. 2006.

[3] B. D. van Veen and K. M. Buckley, "Beamforming: A versatile approach to spatial filtering," IEEE Acoustics, Speech and Signal Magazine, vol. 5, no. 2, pp. 4-24, Apr. 1988.

[4] O. Nadiri and B. Rafaely, "Localization of multiple speakers under high reverberation using a spherical microphone array and the direct-path dominance test," IEEE/ACM Trans. Audio, Speech, Lang. Process., vol. 22, no. 10, pp. 1494-1505, Oct. 2014.

[5] B. Rafaely and D. Kolossa, "Speaker localization in reverberant rooms based on direct path dominance test statistics," in Proc. IEEE Intl. Conf. on Acoustics, Speech and Signal Processing (ICASSP), 2017.

[6] T. Gehrig and J. McDonough, "Tracking multiple speakers with probabilistic data association filters," in Multimodal Technologies for Perception of Humans, ser. Lecture Notes in Computer Science, R. Stiefelhagen and J. Garofolo, Eds. Springer Berlin Heidelberg, 2007, vol. 4122, pp. 137-150.

[7] B. Ristic, S. Arulampalam, and N. Gordon, Beyond the Kalman filter : particle filters for tracking applications. Boston: Artech House, 2004.

[8] B. Rafaely, Fundamentals of Spherical Array Processing, ser. Springer Topics in Signal Processing. Berlin Heidelberg: Springer, 2015.

[9] K. V. Mardia and P. E. Jupp, Directional Statistics. WileyBlackwell, 1999.

[10] A. Doucet, S. Godsill, and C. Andrieu, "On sequential Monte Carlo sampling methods for Bayesian filtering," Statistics and Computing, vol. 10, no. 3, pp. 197-208, 2000.

[11] D. P. Jarrett, E. A. P. Habets, M. R. P. Thomas, and P. A. Naylor, "Simulating room impulse responses for spherical microphone arrays," in Proc. IEEE Intl. Conf. on Acoustics, Speech and Signal Processing (ICASSP), Prague, Czech Republic, May 2011, pp. 129-132.

[12] E. A. Lehmann and R. C. Williamson, "Particle filter design using importance sampling for acoustic source localisation and tracking in reverberant environments," EURASIP J. on Advances in Signal Processing, Jun. 2006.

[13] E. Lehmann and A. Johansson, "Diffuse reverberation model for efficient image-source simulation of room impulse responses," IEEE Trans. Audio, Speech, Lang. Process., vol. 18, no. 6, pp. 1429-1439, Aug. 2010.

[14] J. S. Garofolo, L. F. Lamel, W. M. Fisher, J. G. Fiscus, D. S. Pallett, N. L. Dahlgren, and V. Zue, "TIMIT acousticphonetic continuous speech corpus," Linguistic Data Consortium (LDC), Philadelphia, Corpus LDC93S1, 1993. 